\title{
Immunologic evaluation and validation of methods using synthetic peptides derived from Mycobacterium tuberculosis for the diagnosis of tuberculosis infection
}

\author{
Zaida Araujo ${ }^{1 /+}$, Francesca Giampietro ${ }^{1}$, María de los Angeles Bochichio ${ }^{1}$, Andrea Palacios ${ }^{1}$, \\ Jenifer Dinis', Jaime Isern', Jacobus Henry de Waard², Elsa Rada ${ }^{3}$, Rafael Borges ${ }^{4}$, \\ Carlos Fernández de Larrea ${ }^{5}$, Angel Villasmil' ${ }^{6}$, Magnolia Vanegas ${ }^{7,8}$, \\ Jose Antonio Enciso-Moreno9 ${ }^{9}$, Manuel Alfonso Patarroyo ${ }^{7,8}$
}

\begin{abstract}
${ }^{1}$ Laboratorio de Inmunología de Enfermedades Infecciosas ${ }^{2}$ Laboratorio de Tuberculosis ${ }^{3}$ Laboratorio de Bioquímica ${ }^{4}$ Sección de Epidemiología, Instituto de Biomedicina, Universidad Central de Venezuela, Caracas, Venezuela ${ }^{5}$ Servei d'Hematologia, Hospital Clínic de Barcelona, Barcelona, España ${ }^{6}$ Laboratorio de Genética Molecular, Instituto Venezolano de Investigaciones Científicas, Caracas, Venezuela ${ }^{7}$ Departamento de Biología Molecular, Fundación Instituto de Inmunología de Colombia, Bogotá, Colombia ${ }^{8}$ Departamento de Ciencias Básicas, Universidad del Rosario, Bogotá, Colombia ${ }^{9}$ Unidad de Investigación Médica Zacatecas, Instituto Mexicano del Seguro Social, Zacatecas, México
\end{abstract}

The goal of this study was to demonstrate the usefulness of an enzyme-linked immunosorbent assay (ELISA) for the serodiagnosis of pulmonary tuberculosis (PTB) and extrapulmonary TB (EPTB). This assay used 20 amino acidlong, non-overlapped synthetic peptides that spanned the complete Mycobacterium tuberculosis ESAT-6 and Ag85A sequences. The validation cohort consisted of 1,102 individuals who were grouped into the following five diagnostic groups: 455 patients with PTB, 60 patients with EPTB, 40 individuals with non-EPTB, 33 individuals with leprosy and 514 healthy controls. For the PTB group, two ESAT-6 peptides (12033 and 12034) had the highest sensitivity levels of $96.9 \%$ and $96.2 \%$, respectively, and an Ag85A-peptide (29878) was the most specific (97.4\%) in the PTB groups. For the EPTB group, two Ag85A peptides (11005 and 11006) were observed to have a sensitivity of $98.3 \%$ and an Ag85A-peptide (29878) was also the most specific (96.4\%). When combinations of peptides were used, such as 12033 and 12034 or 11005 and 11006, 99.5\% and 100\% sensitivities in the PTB and EPTB groups were observed, respectively. In conclusion, for a cohort that consists entirely of individuals from Venezuela, a multi-antigen immunoassay using highly sensitive ESAT-6 and Ag85A peptides alone and in combination could be used to more rapidly diagnose PTB and EPTB infection.

Key words: pulmonary tuberculosis - extrapulmonary tuberculosis - synthetic peptide - ESAT-6 antigen - Ag85A antigen

The World Health Organization (WHO) lists tuberculosis (TB) as the most important fatal infection worldwide (WHO 2009). The development of a new diagnostic test for TB infection is an important component of the Global Plan to Stop TB and the WHO Stop TB Strategy. In 2005 alone, an estimated 8.8 million individuals were infected with TB and 1.6 million people died of the disease (WHO 2009). Importantly, less than one half of the total 8.8 million estimated cases were diagnosed as smear-positive; the diagnosis of smear-negative patients has proven to be more challenging. Currently, a definitive diagnosis of both pulmonary TB (PTB) and extrapulmonary TB (EPTB) relies on the time consuming culture of mycobacteria. A vast majority of TB cases occur in developing countries that have limited resources. Rapid, inexpensive diagnostic tests would aid these

Financial support: CDCH/UCV-PI 09-00-6256-2007, LOCTI-022009/04-2010

+ Corresponding author: zaraujogarcia@yahoo.com

Received 8 February 2012

Accepted 7 November 2012 countries in limiting the spread of infection within their communities (WHO 2009). In addition, molecular methods based on nucleic acid amplification to diagnose TB infection are rapid, highly specific and more sensitive than microscopic examination of smears, but are less sensitive compared to culture assays for smear-negative TB cases (Abebe et al. 2007).

Serological tests that rely on the detection of antibodies against Mycobacterium tuberculosis-specific antigens possess several advantages: they are simple, inexpensive and feasible for the diagnosis of TB. Potential $M$. tuberculosis antigens were recently reviewed in a metaanalysis. A total of 254 studies were identified that encompassed nine native proteins, 27 recombinant proteins, 15 lipid-derived antigens and 30 combination antigen targets. These results indicated that highly specific tests frequently exhibited poor sensitivity, which limited the use of these antigens when a single antigen was used in the assay (Steingart et al. 2009). Recently, there has been renewed interest in the development of antibody-based diagnostic assays that utilise multiple antigens to achieve high sensitivity and specificity (Abebe et al. 2007). Many attempts to develop a serologic TB test have been made. These assays need to discriminate active from latent infection, avoid cross-reactivity with Bacillus Calmette- 
Guérin (BCG) or non-tuberculous mycobacteria and be inconsistent and specific in genetically and immunologically diverse populations (Abebe et al. 2007, Ireton et al. 2010). Serodiagnosis of TB has long been the subject of investigation. Several enzyme-linked immunosorbent assays (ELISA) have been attempted and result in large variability depending on whether antigen alone or a pool of antigens was used, the immunoglobulin (Ig) class or subclass measured and the Mycobacterium tuberculosis strain used (Chiang et al. 1997, Turneer et al. 1998, Pottumarthy et al. 2000, Raja et al. 2002, Conde et al. 2004, Mabey et al. 2004, Gupta et al. 2005). This indicates that more optimal antigens have yet to be tested or that there is a differential antibody response as a result of ethnic genetic variation (Lyashchenko et al. 1998, Demkow et al. 2004, Araujo et al. 2008). A successful serodiagnostic test for TB hinges on the capacity of the assay to detect the pauci and multibacillary forms of TB, paediatric TB cases and TB-infected patients coinfected with human immunodeficiency virus (HIV) (Mabey et al. 2004, Tiwari et al. 2007). Many reports have proposed utilising several combinations of antigens or specific antibodies for a serological assay for the diagnosis of TB (Araujo et al. 2004, Kumar et al. 2008, He et al. 2011).

Proteins that are actively secreted during culture on synthetic media are of particular interest. At least eight proteins secreted by $M$. tuberculosis have been isolated and characterised. Of these proteins, the ESAT- 6 and the Ag85 complexes (Ag85c) have been tested to determine their accuracy and consistency for use in a diagnostic assay for TB infection (Wiker \& Harboe 1992, López-Vidal et al. 2004). M. tuberculosis ESAT-6 antigen is small [ $\sim 100$ amino acids (aa), an apparent molecular mass of $6 \mathrm{kDa}$, highly immunogenic and has been shown to induce antibody production. Therefore, it is plausible that the detection of a humoral response to ESAT- 6 may play a fundamental role in the identification of individuals that have been exposed to TB recently and have an increased risk of disease in endemic areas (Harboe 1998, López-Vidal et al. 2004). Often, the Ag85c proteins are the most common proteins identified in M. tuberculosis culture supernatants. The $85 \mathrm{~A}(31 \mathrm{kDa}), 85 \mathrm{~B}(30 \mathrm{kDa})$ and $85 \mathrm{C}(31.5 \mathrm{kDa})$ proteins are encoded by three genes that are located at different sites in the mycobacterial genome. These proteins have extensive cross-reactivity and homology at the aa and gene levels (Wiker \& Harboe 1992). For these three proteins, immunological reactivity has been reported in TB-infected patients and in healthy controls ( $\mathrm{HC}$ ) and the antibody responses to the $85 \mathrm{~B}$ and $85 \mathrm{~A}$ proteins in infected patients were observed to be significantly higher compared to the antibody response to the $85 \mathrm{C}$ protein (Weldingh et al. 2005). Because the diagnoses of EPTB, TB-HIV coinfection, childhood TB and sputum smear-negative PTB are extremely challenging, this study aimed to assess the serodiagnostic potential of synthetic peptide antigens that spanned the complete sequence of M. tuberculosis ESAT-6 and Ag85A proteins for the rapid diagnosis of PTB and EPTB in a majority of patients studied and at an accepted cost-benefit ratio.

\section{PATIENTS, MATERIALS AND METHODS}

Study population - In a previous study, a prospective ELISA trial was performed using 22 different peptides to analyse 95 serum samples (50 adult patients with pulmonary disease and $45 \mathrm{HC}$ ), which allowed the selection of peptides that were observed to have better sensitivity and specificity for validation. In this study, the validation set consisted of a total of 1,102 samples. The experimental group consisted of 455 samples from patients with PTB and 60 samples from patients with EPTB, which included 41 from patients with tuberculous pleural effusion, six from patients with renal TB, eight from patients with lymph node TB, one from a patient with cutaneous TB, two from patients with TB meningitis, one from a patient with miliary $\mathrm{TB}$ and one from a patient with abdominal TB. The control group (CT) consisted of 587 individuals, 514 of whom were HC. Thirty-three individuals were infected with leprosy (LP). Of the total LP samples, 11 were from patients with lepromatous LP, eight from patients with borderline LP, 10 from patients with borderline tuberculoid LP, two from patients with tuberculoid LP and two from patients with borderline LP (the CT group includes the HC and LP groups). Of the CT group, 40 samples were obtained from patients with non-EPTB (NEPTB), including 14 from patients with non-tuberculous pleural effusion or malignant pleural effusions, 11 from patients with parapneumonic exudates, 10 from patients with transudates and five from patient miscellaneous exudates (the NEPTB group).

Inclusion criteria for individuals with progressive $T B$ prior to treatment - Similar to criteria that have been previously published (Kudoh \& Kudoh 1974), PTB was diagnosed using any one of the following criteria: (i) $M$. tuberculosis staining positive for acid-fast bacilli (ZiehlNeelsen dye) and isolated from the sputum by culture and (ii) non-stainable acid-fast bacilli in the smear but M. tuberculosis isolated from the sputum by culture.

All patients who were included in the study were undergoing thoracentesis for diagnostic purposes by the Department of Pneumology of the Caracas Vargas Hospital. A diagnosis of TB pleural effusions was made in these patients. Pleural fluid and biopsy samples that were simultaneously obtained from patients after thoracentesis were subjected to cytological and microbiological (including mycobacteriological) culture and cytokine assays. Pleural biopsies were performed only when the acid-fast bacillus staining (smear) or the culture of pleural fluid to detect M. tuberculosis were negative. As recently suggested by the National TB Program (Fernández de Larrea et al. 2011), EPTB was diagnosed by the following criteria: (i) M. tuberculosis isolated from pleural effusion by culture, (ii) pleural biopsy indicating granulomatous inflammation in addition to positive acid-fast bacilli staining, (iii) pleural biopsy indicating granulomatous inflammation, negative acid-fast bacilli stain and either positive sputum culture for M. tuberculosis or good radiographic response to anti-TB treatment and (iv) no histological or bacteriological confirmation, but alternative diagnoses were excluded in addition to good clinical and radiographic response to anti-TB treatment. 
Inclusion criteria for controls - HCs living in Caracas consisted of laboratory staff and medical student volunteers who were negative for HIV and active TB infections, as determined by blood tests and X-rays, respectively. Individuals with malignant pleural effusions were diagnosed by pleural effusion cytology or by biopsy. Miscellaneous pleural effusions were determined to be those not linked to infection or malignant disease. Patients with LP were clinically and histologically diagnosed according to the classification of Ridley and Jopling (1966). The CT groups included individuals with or without the presence of BCG scars. The tuberculin skin test (TST) was performed according to international standards and the existing norms of the Venezuelan Program of Tuberculosis Control (Arnadottir et al. 1996, MSDS 2006) for all of the individuals utilised for this study. Two tuberculin units of M. tuberculosis RT-23 purified protein derivative (PPD) from the Statens Serum Institute in Copenhagen, Denmark were used for the TST test. An individual was determined to be $\mathrm{TST}^{+}$if an induration with a diameter $\geq 10 \mathrm{~mm}$ was observed $48 \mathrm{~h}$ post-test. The CT groups included TST ${ }^{+}$and TST- $^{-}$individuals.

Treatment was initiated for all patients with microbiological evidence that was suggestive of TB infection and for whom positive bacilloscopy or culture was determined. Clinical and nutritional follow-up was performed for six months after anti-TB drug treatment to evaluate the treatment regimen. All of the patients were confirmed to be HIV-negative. Individuals with or without the presence of BCG scars were included in the study.

Ethics - This study was approved by the Ethical Committee of the Biomedicine Institute and the Caracas Vargas Hospital (protocol CDCH-6256-06/LOCTI02-2009/12/03/08). Written informed consent was provided and voluntarily signed by all individuals enrolled in the study.

Synthetic peptide, recombinant ESAT-6 (rESAT-6) and $A g 85 \mathrm{c}$ - Because it has been shown that 3-5 aa contribute strongly to the antigen-antibody binding within a linear B-cell epitope (Cunningham \& Wells 1993), up to six antibody-binding sites are expected in a single 20 -mer peptide. Twenty aa-long, non-overlapping synthetic peptides that corresponded to the full lengths of ESAT- 6 and Ag85A were synthesised and used as single-peptide antigens (Fundación Instituto de Immunologia de Colombia, Bogotá, Colombia). The following 5 ESAT-6-peptides were synthesised: P1 (12033, 1-20; MTEQQWNFAGIEAAASAIQG), P2 (12034, 21-40; NVTSIHSLLDEGKQSLTKLA), P3 (12035， 41-60; AAWGGSGSEAYQGVQQKWDA), P4 (12036, 61-80; TATELNNALQNLARTISEAG) and P5 (12037, 7695; ISEAGQAMASTEGNVTGMFA). There were 17 Ag85A peptides synthesised as follows: P1 (29878, 1-22; MQLVDRVRGAVTGMSRRLVVGAY), P2 (29879, 2343; VGAALVSGLVGAVGGTATAGAY), P3 (10993, 4463; FSRPGLPVEYLQVPSPSMGR), P4 (10994, 64-83; DIKVQFQSGGANSPALYLLD), P5 (10995, 84-104; GLRAQDDFSGWDINTPAFEWY), P6 (10996, 104-123; YDQSGLSVVMPVGGQSSFYS), P7 (10997, 124-143; DWYQPACRKAGCQTYKWETF), P8 (10998, 144-163;
LTSELPGWLQANRHVKPTGSY), P9 (10999, 164-182; AVVGLSMAASSALTLAIYH), P10 (11000, 183-203; PQQFVYAGAMSGLLDPSQAMG), P11 (29880, 204223; PTLIGLAMGDAGGYKASDMW), P12 (11002, 224-243; GPKEDPAWQRNDPLLNVGKLY), P13 (11003, 244-263; IANNTRVWVYCGNGKPSDLG), P14 (11004, 264-283; GNNLPAKFLEGFVRTSNIKFY), P15 (11005, 284-303; QDAYNAGGRHNGVFDFPDSG), P16 (11006, 304-322; THSWEYWGAQLNAMKPDLQ) and P17 (29881, 320-338; DLQRALGATPNTGPAPQGAY). Peptides were synthesised based on the aa sequences of M. tuberculosis ESAT-6 and Ag85A using a solid-phase multiple-peptide system (Merrifield 1963, Tam \& Heath 1983, Houghten 1985). rESAT-6) antigen was obtained from Statens Serum Institute (Copenhagen, Denmark). The Ag85c was procured from the Mycobacteria Research Laboratories (Colorado State University, USA).

Serologic assays - The serum IgG levels that were reactive against rESAT-6, ESAT-6 peptides and the Ag85c and $\mathrm{Ag} 85 \mathrm{~A}$ peptides were determined by an ELISA as previously described (Araujo et al. 2008). Briefly, serum was isolated from venous blood obtained from $\mathrm{HC}$ individuals and patients. Ninety-six-well microtitre plates (Immunolon, Birmingham, UK) were coated with the following (1 $\mu \mathrm{g} / \mathrm{well})$ : rESAT- 6 antigen and the Ag85c or the ESAT-6 and Ag85A synthetic peptides. Immunoenzymatic assays were performed and standardised for the measurement of reactive antibodies (IgG). After standardisation, serum samples were diluted at 1:200. After incubation, plates were then incubated with an anti-IgG peroxidase-conjugated monoclonal antibody (Promega Corporation, USA). Plates were washed four times and a substrate solution consisting of citrate buffer ( $\mathrm{pH} 5.0$ ), $\mathrm{H} 2 \mathrm{O} 230 \%$ and 10 mg ortho-phenylenediamine dihydrochloride (Sigma-Aldrich) was added. The plates were incubated for $6 \mathrm{~min}$ at room temperature and colour development was measured using an ELISA microplate reader at $492 \mathrm{~nm}$.

Statistical analysis - Receiver operating characteristic (ROC) curves were utilised to analyse the diagnostic information of each method by comparing the area under the curve (AUC), cut-off values, sensitivity, specificity, predictive values and the likelihood ratio. Comparisons of average age between groups were performed using the Student's $t$ test and Fisher's exact test to compare differences between individuals who were positive and negative for the TST. Differences between isotype reactivities were compared between groups using the Mann-Whitney $U$ test. A scattergram was plotted using GRAPHPAD Prism v5.02 software (Trial version, GraphPad Software, Inc, San Diego, CA, USA). Statistically significant differences were those determined to have a $\mathrm{p}$ value $\leq 0.05$.

\section{RESULTS}

Gender, age and BCG vaccination - There was a statistically significant difference in gender for the PTB group, with $267 / 455$ being female and $188 / 455$ being male $(p<0.0001)$. A significant difference was also observed for the EPTB group, with 45/60 being female and 15/60 being male $(p<0.0001)$ (Table I). Statistically significant 
differences also existed between the numbers of females and males in the HC group, with 200/514 being female and $313 / 514$ being male $(\mathrm{p}<0.0001)$ and for the NEPTB group, with $25 / 40$ being female and $15 / 40$ being male ( $p$ $<0.04$ ) (Table I). There was no statistically significant difference in the average ages of individuals between the patient and CT groups (Table I). Both patients and $\mathrm{HC}<$ 20 years of age were more likely to have received BCG vaccination as either neonates and/or during childhood as part of the increasingly effective Venezuelan National BCG Vaccination Program (data not shown).

Bacteriological and immunological studies - Table I provides information about the bacteriological and tuberculin skin tests performed in this study. Either smear alone or smear in addition to culture analysis were performed for HC, NEPTB-infected patients and LP patients (Table I). The skin reactivity test was performed to study delayed-type hypersensitivity. Greater than $10 \mathrm{~mm}$ reactions were considered to be positive. Statistically significant differences in the percentages of patients that were $\mathrm{TST}^{+}$were observed between the PTB and EPTB groups $(p<0.0001)$ and between the HC group and both the NEPTB $(\mathrm{p}<0.0001)$ and LP groups $(\mathrm{p}<0.002)$ (Table I).

Optical density $(O D)$ distribution and ROC analysis The analysis of ROC curves and the mean \pm the standard deviations of OD 492 distributions are shown in Figs 1, 2. Evaluation of the IgG reactivity against peptides 12033, 12034 and 12037 revealed significantly higher anti-peptide $\mathrm{IgG}$ reactivities in the PTB group $(0.436 \pm 0.267$, $0.500 \pm 0.292$ and $0.504 \pm 0.283$ ) when compared to the CT group $(0.338 \pm 0.240,0.391 \pm 0.256$ and $0.391 \pm 0.197)$ $(\mathrm{p}<0.0001)$ (Fig. 1A). Cut-off values for peptides 12033 $[\mathrm{AUC}=0.554 ; 95 \%$ confidence interval $(\mathrm{CI}), 0.448$ 0.657 ], 12034 (AUC $=0.688 ; 95 \%$ CI, 0.584-0.779) or $12037(\mathrm{AUC}=0.529 ; 95 \% \mathrm{CI}, 0.423-0.633)$ were $>0.113$, $>0.143$ and $>0.716$, respectively (Fig. 1A). For Ag85A, the IgG reactivities against peptides 10994 and 10998 were significantly higher for the PTB group $(0.471 \pm 0.265$ and $0.453 \pm 0.318)$ when compared to the CT group $(0.402 \pm$
0.252 and $0.343 \pm 0.250)(\mathrm{p}<0.0001)$ (Fig. 1B). Cut-off values for peptides $29878((\mathrm{AUC}=0.526 ; 95 \% \mathrm{CI}, 0.420$ 0.630 ), 10994 ( $\mathrm{AUC}=0.692 ; 95 \% \mathrm{CI}, 0.588-0.783$ ) and $10998(\mathrm{AUC}=0.591 ; 95 \%$ CI, 0.485-0.691) were $\leq 0.083$, $>0.401$ and $>0.261$, respectively (Fig. 1B).

The IgG reactivity against ESAT-6 peptide 12035 was significantly higher in the EPTB group $(0.496 \pm 0.293)$ when compared to the NEPTB group $(0.372 \pm 0.245)(\mathrm{p}<$ 0.04) (Fig. 2A). Cut-off values for peptides 12033 (AUC $=0.520 ; 95 \% \mathrm{CI}, 0.386-0.652), 12035(\mathrm{AUC}=0.662$; $95 \% \mathrm{CI}, 0.527-0.780)$ and $12037(\mathrm{AUC}=0.572 ; 95 \% \mathrm{CI}$, $0.436-0.700$ ) were $>0.276,>0.331$ and $>0.223$, respectively (Fig. 2A). Ag85A peptide 11006 was observed to have a significantly higher anti-peptide $\operatorname{IgG}$ response in the EPTB group $(0.384 \pm 0.148)$ when compared to the NEPTB group $(0.297 \pm 0.183)(\mathrm{p}<0.002)$. In contrast, the $\operatorname{IgG}$ reactivity was significantly higher against peptide 10997 in the NEPTB group $(0.451 \pm 0.241)$ when compared to the EPTB group $(0.306 \pm 0.140)(\mathrm{p}<0.001)$. Cut-off values for peptides 10997 (AUC $=0.620 ; 95 \%$ : CI; 0.484-0.743), 11004 (AUC $=0.523$; 95\% CI, 0.389$0.655), 11005$ (AUC $=0.514 ; 95 \%$ CI, 0.381-0.647), $11006(\mathrm{AUC}=0.529 ; 95 \% \mathrm{CI}, 0.395-0.660), 29878(\mathrm{AUC}$ $=0.551 ; 95 \% \mathrm{CI}, 0.416-0.681)$ and $29880(\mathrm{AUC}=0.529$; $95 \% \mathrm{CI} ; 0.395-0.660)$ were $\leq 0.526, \leq 0.496, \leq 0.741,>$ $0.156, \leq 0.244$ and $>0.214$, respectively (Fig. $2 B$ ).

For the Ag85c and rESAT- 6 antigens, IgG reactivity was significantly higher against both antigens in the PTB group $(0.714 \pm 0.308$ and $0.435 \pm 0.203)$ when compared to the CT group $(0.326 \pm 0.186$ and $0.192 \pm 0.113)$ $(p<0.0001)$ (data not shown). Cut-off values of $>0.410$ and $>0.248$ were observed for the $\mathrm{Ag} 85 \mathrm{c}(\mathrm{AUC}=0.872$; $95 \% \mathrm{CI}, 0.761-0.944)$ and rESAT-6 $(\mathrm{AUC}=0.874 ; 95 \%$ CI, 0.763-0.945) antigens, respectively.

Sensitivity and specificity - The sensitivities and specificities of the different ELISA tests for the PTB and EPTB groups are illustrated in Table II. For the PTB group, the sensitivity ranged from $9 \%$ (peptide 12037) to $96.9 \%$ (peptide 12033) and the specificity ranged

TABLE I

Age and gender characteristics and immunological and bacteriological markers

\begin{tabular}{|c|c|c|c|c|c|}
\hline Marker & РTB & ЕРТВ & $\mathrm{HC}$ & NEPTB & LP \\
\hline Age (years) & $38.6 \pm 24.8$ & $34.7 \pm 16.9$ & $35.3 \pm 21.2$ & $51 \pm 19.4$ & $28 \pm 21.4$ \\
\hline Male (\%) & 41.3 & 25 & 60.9 & 37.5 & 42.4 \\
\hline $\mathrm{TST}^{+}(\%)$ & $93.7^{a}$ & $50^{b}$ & $42.1^{c}$ & $17.5^{d}$ & $60.6^{e}$ \\
\hline Smear $^{+}(\%)$ & 64 & 20 & 0 & 0 & 0 \\
\hline Smear $^{+}+$culture ${ }^{+}(\%)$ & 100 & 70 & 0 & 0 & 0 \\
\hline
\end{tabular}

$a, b: \mathrm{p}<0.0001 ; c, d: \mathrm{p}<0.0001 ; d, e: \mathrm{p}<0.002$. Age results are shown as mean \pm standard deviation. Serum samples were collected from adult patients with a confirmed tuberculosis (TB) diagnosis according existing norms in the Venezuelan Program of Tuberculosis Control. Volunteers in whom human immunodeficiency virus and active TB were ruled out by blood tests and $\mathrm{X}$-rays, respectively. Tuberculin skin test (TST) ${ }^{+}$status was defined for an induration with a diameter equal or superior to 10 $\mathrm{mm}$ after $48 \mathrm{~h}$ of the test. There was a statistically significant differences between feminine and masculine groups in PTB (p $<0.0001)$, EPTB $(\mathrm{p}<0.0001)$, HC $(\mathrm{p}<0.0001)$ and NEPTB $(\mathrm{p}<0.04)$ and also between $a$ and $b, c$ and $d$ and $d$ and $e$. EPTB: extrapulmonary TB; HC: healthy controls; LP: leprosy; NEPTB: non-EPTB. 
from 14.1\% (peptide 12033) to 97.4\% (peptide 29878) (Table II). For the EPTB group, the sensitivity ranged from $21.6 \%$ (peptide 29878) to $98.3 \%$ (peptides 11005 and 11006 ) and the specificity ranged from $15 \%$ (peptide 11005 ) to $96.4 \%$ (peptide 29878) (Table II).

Of all of the antigen and combinations studied, four tests allowed the detection of infection in the largest number of patients with high levels of sensitivity. For the PTB and EPTB groups, combinations of two ESAT6-peptides (peptides 12033 and 12034) or two Ag85Apeptides (peptides 11005 and 11006) yielded sensitivities of $99.5 \%$ and $100 \%$, respectively, but respective specificity levels dropped to $10.2 \%$ and $2.5 \%$ (Table II). Tests that included recombinant antigens, such as Ag85c and rESAT-6, were observed to have sensitivities of $85 \%$ and $95 \%$, respectively. These sensitivities were lower compared to the use of the peptide combinations 12033/12034 or 11005/11006 (Table II).

\section{DISCUSSION}

This study was focused on the use of ESAT-6 and Ag85A synthetic peptides to improve the early diagnosis of patients infected with PTB and EPTB. Initial ELISA screens identified 13 peptides out of 22 that had acceptable accuracies for diagnosing TB infection. These highly sensitive and specific peptides were further studied and validated in a larger panel of 515 patient samples and 587 HC serum samples. For the PTB group, the overall sensitivities of the six immunological peptides ranged from 9.02-96.9\%. Two peptides exhibited high sensitivities: peptide $12033(96.9 \%)$ and peptide 12034 (96.2\%). For the EPTB group, the overall sensitivities of the seven peptides that were validated ranged from $21.6-98.3 \%$. Two peptides, 11005 and 11006, had high sensitivities (98.3\%) and peptide 29878 exhibited even higher specificity (specificities of $97.4 \%$ and $96.4 \%$ for the PTB and EPTB groups, respectively). Moreover, combinations of peptides indicated that ESAT-6-peptides 12033/12034 and Ag85A-peptides 11005/11006 exhibited maximum sensitivities of $99.5 \%$ and $100 \%$, respectively, which allowed the detection of the largest number of PTB and EPTB patients. The latter results, which demonstrated high IgG reactivities against synthetic peptides that covered the complete sequences of ESAT-6 and Ag85A, are in agreement with a study indicating that the immunogenicity of synthetic peptides is a result of the peptide's capacity to elicit anti-peptide antibodies that cross-react with the cognate, intact protein (Van Regenmortel 2001).

For statistical analysis, the narrower the CI, the more precise the value is for multiple comparisons. Within this context, the sensitivities of five tests, including the 12033 and 12034 peptide tests, the rESAT- 6 antigen, Ag85c antigen and the 12033/12034 combination peptide test, were equivalent because the CIs overlapped. The combination test was observed to have the highest sensitivity at 99.5\%. The 10994, 10998, 12037 and 29878 peptide tests were also equivalent; peptide 29878 exhibited the highest specificity at $97.4 \%$. Because LP is also a mycobacterial infection, the specificity of the assays could be affected by cross-reactivity with Mycobacterium leprae. However, it was demonstrated that the specificity was not affected by including serum from patients with LP in this study.
To control the spread of PTB, effective vaccination and accurate early diagnosis of the disease are critical. It has been estimated that a rapid and widely available diagnostic test with $85 \%$ sensitivity and $95 \%$ specificity would result in 400,000 fewer deaths each year and would reduce the spread of TB (Ireton et al. 2010). These sensitivities and specificities are lower than those reported in the tests in this study using peptides 12033 (96.9\%), $12034(96.2 \%)$ and $29878(97.4 \%)$ and the combination of peptides 12033/12034 (99.5\%). The diagnosis of TB-HIV coinfection, childhood TB and sputum smear-negative PTB infection still poses serious challenges. The morphological identification of acid-fast bacilli in sputum smears is a faster but less-sensitive method compared to culture because it requires a higher number of organisms (roughly $50 \%$ of cases are positive overall) and is labourintensive (Gupta et al. 2005). This underscores the need
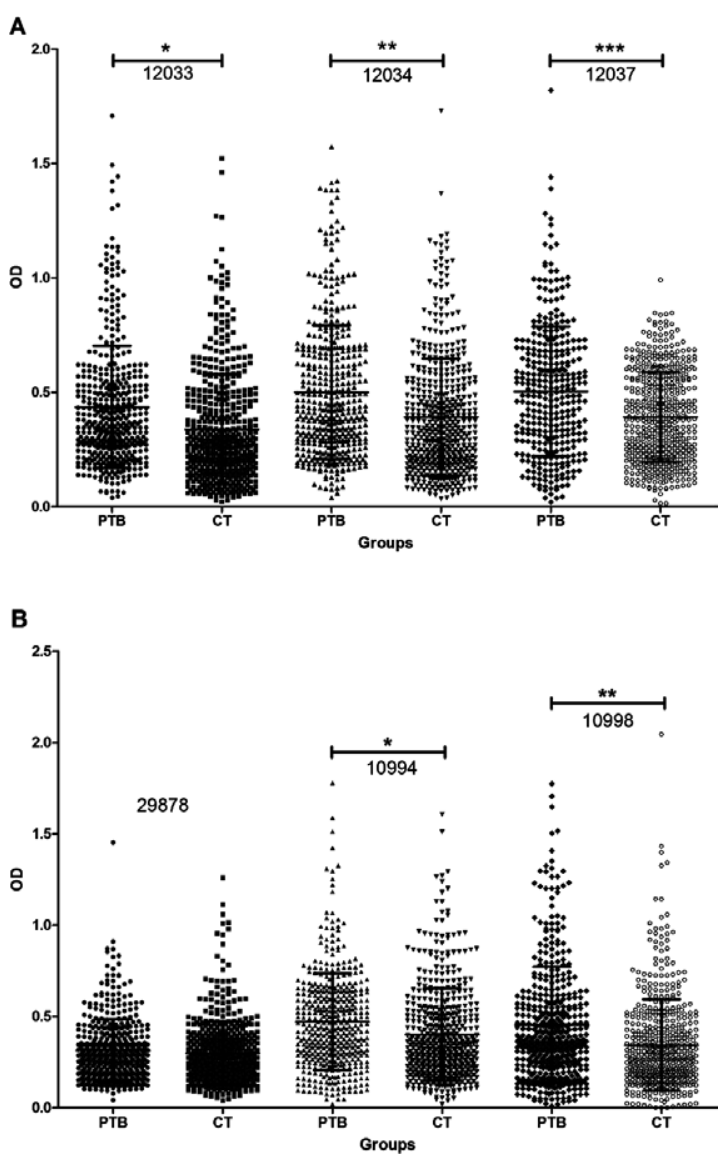

Fig. 1: analysis of receiver operating characteristic and comparison of the optical density (OD) distribution are shown between patient with pulmonary tuberculosis (PTB) and control group (CT) (composed by health control and leprosy groups). A: antibody reactivity to ESAT-6 peptides: 12033 peptide [PTB $(\bullet)$ and CT $(\boldsymbol{\bullet})$ ], cut-off $(\leftrightarrow)>0.113$, 12034 peptide [PTB $(\mathbf{\Lambda})$ and CT $(\boldsymbol{\nabla})$ ], cut-off $(\leftrightarrow)>0.143,12037$ peptide [PTB $(\diamond)$ and CT $(\mathrm{o})$ ], cut-off $(\leftrightarrow)>0.716 . *, * *, * * *$ : the significance between PTB and CT groups, $\mathrm{p}<0.0001$; B: antibody reactivity to Ag85A peptides: 28978 peptide [PTB $(\bullet)$ and CT $(\boldsymbol{\bullet})$ ], cut-off $(\leftrightarrow)$ $<=0.083,10994$ peptide [PTB $(\mathbf{\Delta})$ and CT $(\boldsymbol{\nabla})$ ], cut-off $(\leftrightarrow)>0.401$, 10998 peptide [PTB $(\diamond)$ and CT $(\mathrm{o})$ ], cut-off $(\leftrightarrow)>0.261 . *, * *$ : the significance between PTB and CT groups, $\mathrm{p}<0.0001$. 
for a rapid, sensitive diagnostic test that could confirm TB infection and help in TB control efforts. In our study, the majority of sputum smear-negative patients with PTB were observed to have antibodies that were highly sensitive against ESAT-6 peptides (12033 and 12034). Additionally, the sensitivities of these peptides, especially in combination, were higher compared to the classical smear assay (sensitivity, 64\%). Recently, monoclonal antibodies were generated against the M. tuberculosis-specific antigen ESAT-6, resulting in an ESAT-6-specific ELISA that yielded a specificity of $100 \%$ and a sensitivity of $95.4 \%$ (Feng et al. 2011). These data correlate well with our findings when using this antigen. However, the sensitivity achieved using rESAT-6 was lower (95\%)

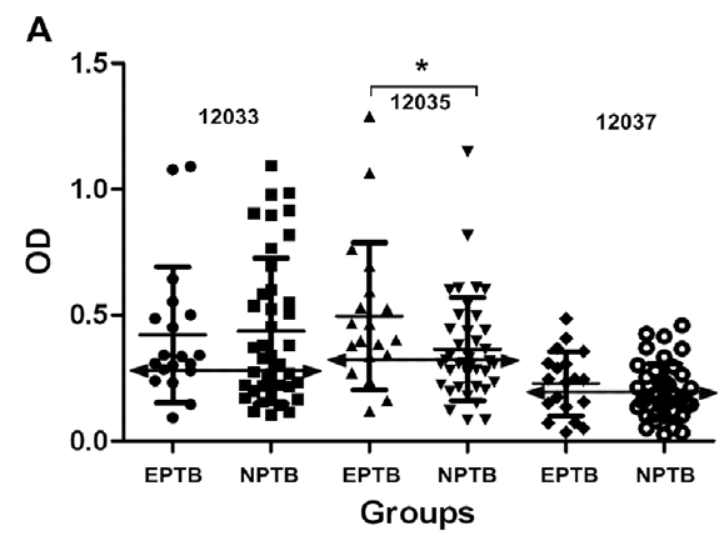

B

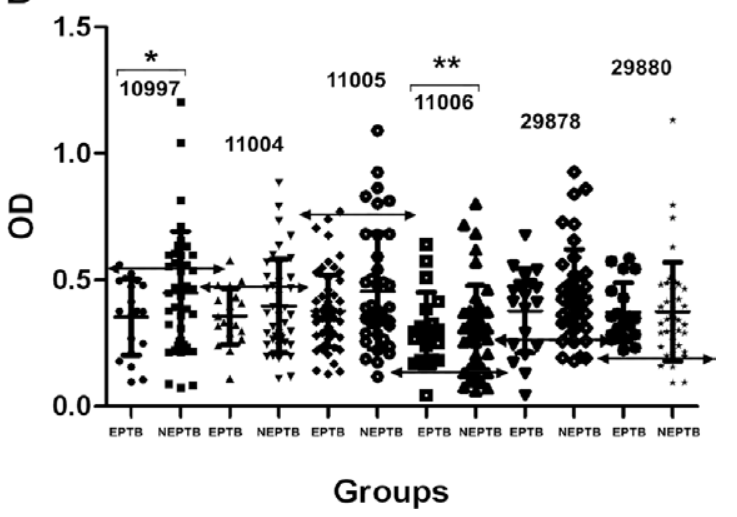

Fig. 2: analysis of receiver operating characteristic and comparison of the optical density (OD) distribution are shown between patients with extrapulmonary tuberculosis (EPTB) and non-EPTB. A: antibody reactivity to ESAT- 6 peptides: 12033 peptide [EPTB $(\bullet)$ and NEPTB $(\mathbf{\bullet})$ ], cut-off $(\leftrightarrow)>0.276,12035$ peptide [EPTB $(\boldsymbol{\Delta})$ and NEPTB $(\boldsymbol{\nabla})$ ], cut-off $(\leftrightarrow)>0.331,12037$ peptide [EPTB $(\bullet)$ and NEPTB (o)], cutoff $(\leftrightarrow)>0.223$. Asterisk means the significance between EPTB and NEPTB groups, $\mathrm{p}<0.04$; $\mathrm{B}$ : antibody reactivity to Ag85A peptides: 10997 peptide [EPTB $(\bullet)$ and NEPTB $(\bullet)$ ], cut-off $(\leftrightarrow)<=0.526$, 11004 peptide [EPTB $(\mathbf{\Delta})$ and NEPTB $(\boldsymbol{\nabla})$ ], cut-off $(\leftrightarrow)<=0.496$, 11005 peptide [EPTB $(\bullet)$ and NEPTB $(\mathrm{o})$ ], cut-off $(\leftrightarrow)<=0.741$, 11006 peptide [EPTB $(\square)$ and NEPTB $(\Delta)]$, cut-off $(\leftrightarrow)>0.156$, 29878 peptide [EPTB $(\nabla)$ and NEPTB $(\diamond)$ ], cut-off $(\leftrightarrow)<=0.244$, 29880 peptide [EPTB $(\bullet)$ and NEPTB $(\star)$ ], cut-off $(\leftrightarrow)>0.214 . *$ : the significance between NEPTB and EPTB groups, $p<0.001$; **: the significance between EPTB and NEPTB groups, $\mathrm{p}<0.002$. compared to the ESAT- 6 peptides. Thus, we developed a new diagnostic test for PTB using ESAT-6 peptides that is acceptable, easy and cost-effective. Conversely, very limited information about the difference in the humoral immune responses against each of the three components of the native Ag85c is available because these antigens are difficult to purify in large amounts by standard biochemical techniques. A monoclonal antibody was also generated against the Ag85c and the antigen was found in the sputum of patients with PTB (Wallis et al. 1998) and in patients with EPTB. A study performed using cerebrospinal fluid indicated that an indirect ELISA test using monoclonal antibodies against the Ag85c yielded $82 \%$ sensitivity and $86 \%$ specificity (Kashyap et al. 2007). In this study, the $\mathrm{Ag} 85 \mathrm{c}$ test $(\mathrm{Ag} 85 \mathrm{c})$ reached $85 \%$ sensitivity and $77.5 \%$ specificity and the Ag85A peptide tests achieved better sensitivity and specificity when compared to Ag85c: peptides 11005 and 11006 had a sensitivity of $98.3 \%$ for the EPTB group and peptide 29878 exhibited specificities of $97.4 \%$ and $96.4 \%$, for the PTB and EPTB groups, respectively.

After statistical analysis for the EPTB group, the sensitivities of the tests using peptides 11005, 11006, 29880, 10997, 11004, 12033 and 12035 and the 11005/11006 combination were not statistically different. Peptide test 12035 was observed to have the highest sensitivity $(100 \%)$. After statistical analysis of the specificity, no statistically significant differences were observed for peptides 11005 , 11006, 29880, 10997, 11004, 12033, 12035, 12037 and 29878. Peptide 29878 was observed to have the highest specificity $(96.4 \%)$. These data are promising and allow us to suggest that these peptide tests are useful as complementary tests to exclude patients who are not infected and to rapidly diagnose patients with EPTB. However, difficulties in diagnosing EPTB exist. Sample cultures take several weeks and in many cases, the diagnosis takes a long time (Honore et al. 2001). This results in many patients being lost to follow-up. Other tests, such as biopsy, the polymerase chain reaction-based methods and the identification of enzymes, such as adenosine deaminase and cytokines, such as interferon- $\gamma($ IFN- $\gamma$ ) and tumour necrosis factor- $\alpha$, in the serum have been designed to improve diagnosis. However, these procedures are not performed routinely in many developing countries (Valdes et al. 1993). For the diagnosis of EPTB, IFN- $\gamma$ identification in the pleural effusion for TB pleuritis patients has emerged as an alternative to the identification of IFN- $\gamma$ in the serum because pleural IFN- $\gamma$ determination is a sensitive and specific test for the diagnosis of TB pleuritis. The measurement of cytokine levels in pleural effusion was found to be useful in the diagnosis of the patients with EPTB because the level of pleural IFN- $\gamma$ in patients with EPTB was high and had elevated diagnostic usefulness (Aoe et al. 2003, Fernández et al. 2011). Therefore, the combined analysis of cytokine levels and humoral reactivity against the combination $11005 / 11006$ peptide test should be analysed in parallel with clinical tests. This process could represent a fast and effective diagnostic algorithm for improving diagnosis prior to bacterial culture. Treatment for TB infection could be initiated more 
rapidly or cytological and pleural biopsy could be considered. In addition, while the IFN- $\gamma$ release assay (IGRA), which detects IFN- $\gamma$ secreted by T-cells in response to M. tuberculosis antigens, has emerged as a specific, alternative test, it is important to investigate whether the IGRA test could be of increased value in the diagnosis of EPTB as compared to the pleural IFN- $\gamma$ test. Utilising a combination of tests, such as a serological test and the IFN- $\gamma$ test, may be complementary and could improve the sensitivity or specificity of EPTB diagnosis.

Few studies have been reported that utilise an ELISA for the detection of antibodies that are reactive against M. tuberculosis synthetic peptides. A study that characterised the ESAT-6 B-cell epitopes for use in an ELISA supports the use of ESAT-6 synthetic peptides instead of rESAT-6 (López-Vidal et al. 2004). Because of the high sensitivities observed, both with ESAT-6 peptides and with Ag85A peptides, we suggest that using these peptides in ELISA tests could be promising for the diagnosis of PTB and EPTB infection.

Our results indicated that patients with PTB reacted significantly to residues in the hydrophilic region of the ESAT-6 protein (Harboe 1998). This hydrophilic region appears in two of the five ESAT-6 peptides that were tested: the 12033 and 12034 peptides, which consisted of residues 1-20 (MTEQQWNFAGIEAAASAIQG) and 21-40 (NVTSIHSLLDEGKQSLTKLA), respectively. The latter did not correlate with findings that were reported in Mexican patients infected with TB: high IgG reactivity against residues 61-80 (TATELNNALQNLARTISEAG) was observed (López-Vidal et al. 2004). Therefore, these data could indicate that heterogeneity in the antibody response between populations could exist in response to antigen. With regard to Ag85A peptides, patients with EPTB reacted significantly against Ag85A peptides (11005 and 11006) that contained residues 284-303 (QDAYNAGGRHNGVFDFPDSG) and 304-322 (THSWEYWGAQLNAMKPDLQ). The majority of epitopes are discontinuous epitopes that typically contain segments of contiguous residues that are able to bind antibody (Van Regenmortel 2001). This was observed for four of the 22 synthetic peptides that were analysed in this study: ESAT-6 peptides 12033 and 12034 for PTB infection and Ag85A peptides 11005 and 11006 for EPTB infection. Thus, in the case of EPTB compared to PTB, heterogeneity in the recognition of antigen exists between Venezuelan individuals infected with TB. This heterogeneity must be taken into account to develop antibodies for use in a diagnostic serological test. Recently, many studies reported that combinations

TABLE II

Analysis of receiver operating characteristics curves of the tests selected for validation

\begin{tabular}{|c|c|c|c|c|c|c|c|}
\hline Tests & $\begin{array}{c}\text { Sensitivity } \\
(\%)\end{array}$ & $\mathrm{CI}$ & $\begin{array}{c}\text { Specificity } \\
(\%)\end{array}$ & $\mathrm{CI}$ & $\mathrm{LR}^{+}$ & $\mathrm{LR}^{-}$ & PPV/NPV \\
\hline \multicolumn{8}{|c|}{ РTB } \\
\hline $12033 / 12034$ & 99.5 & $98.6 / 99.9$ & 10.2 & $8.1 / 128$ & 1.09 & 0.18 & $49.4 / 86.4$ \\
\hline 12033 & 96.9 & $94.8 / 98.4$ & 14.1 & $11.0 / 17.6$ & 1.13 & 0.21 & $49.8 / 84.2$ \\
\hline 12034 & 96.2 & $93.8 / 97.8$ & 16.5 & $13.2 / 20.2$ & 1.15 & 0.23 & $50.3 / 83.3$ \\
\hline rESAT-6 & 95 & $75.1 / 99.2$ & 77.5 & $61.5 / 89.1$ & 4.22 & 0.06 & $67.9 / 96.9$ \\
\hline Ag85c & 85 & $62.1 / 96.6$ & 77.5 & $61.5 / 90.1$ & 3.78 & 0.19 & $65.4 / 91.2$ \\
\hline 10994 & 47.2 & $30.4 / 64.5$ & 94.8 & $85.6 / 98.9$ & 9.13 & 0.56 & $85.0 / 74.3$ \\
\hline 10998 & 38.9 & $23.2 / 56.5$ & 94.8 & $85.6 / 98.9$ & 7.52 & 0.64 & $82.4 / 71.4$ \\
\hline 29878 & 27.8 & $14.2 / 45.2$ & 97.4 & $95.8 / 98.7$ & 1.02 & 0.18 & $67.6 / 97.2$ \\
\hline 12037 & 9.02 & $6.40 / 12.2$ & 94.9 & $92.5 / 96.7$ & 1.78 & 0.98 & $61.0 / 54.2$ \\
\hline \multicolumn{8}{|c|}{ ЕРТВ } \\
\hline $11005 / 11006$ & 100 & $91.5 / 100.0$ & 2.5 & $0.3 / 8.8$ & 1.09 & 0.99 & $60.5 / 50.0$ \\
\hline 11005 & 98.3 & 91.0/99.9 & 15 & $5.7 / 29.8$ & 1.01 & 0.67 & $60.8 / 66.6$ \\
\hline 11006 & 98.3 & 91.0/99.9 & 30 & $16.6 / 46.5$ & 1.01 & 0.67 & $60.2 / 50.0$ \\
\hline 29880 & 96.6 & $88.4 / 99.5$ & 20 & $9.1 / 35.7$ & 1.02 & 0.67 & $60.4 / 50.0$ \\
\hline 10997 & 94.7 & 73.9/99.1 & 27.5 & $14.6 / 43.9$ & 1.31 & 0.19 & $38.3 / 91.7$ \\
\hline 11004 & 94.7 & 73.9/99.1 & 32.5 & $24.9 / 56.7$ & 1.58 & 0.13 & $42.9 / 94.1$ \\
\hline 12033 & 78.9 & $54.4 / 93.8$ & 45 & $29.3 / 61.5$ & 1.44 & 0.47 & $40.4 / 81.8$ \\
\hline 12035 & 78.9 & $54.4 / 93.8$ & 57.5 & $40.9 / 72.9$ & 1.86 & 0.37 & $46.9 / 85.2$ \\
\hline 12037 & 57.9 & $33.5 / 79.7$ & 70 & $53.5 / 83.4$ & 1.93 & 0.60 & $47.8 / 77.8$ \\
\hline 29878 & 21.6 & $12.0 / 34.2$ & 96.4 & $81.2 / 96.5$ & 1.25 & 0.58 & $46.4 / 34.7$ \\
\hline
\end{tabular}

tests showing the best sensitivity and/or specificity were selected for validation. Synthetic peptides derived from the sequence of ESAT-6 and Ag85A, as well as recombinant ESAT-6 (rESAT-6) and Ag85complex (Ag85c) were tested. CI: confidence interval; EPTB: extrapulmonary tuberculosis; LR: likelihood ratio; NPV: negative predictive value; PPV: positive predictive value. 
of M. tuberculosis antigens or an optimal multi-antigen cocktail should be designed to account for the heterogeneity of antibody responses and to achieve the highest sensitivity (Ireton et al. 2010, He et al. 2011). As mentioned previously, when combinations of peptides were analysed, it was found that the peptides used in combination offered improved sensitivity. Combinations of ESAT-6 peptides 12033 and 12034 and Ag85A peptides 11005 and 11006 were able to achieve $99.5 \%$ and $100 \%$ sensitivity, respectively.

Finally, this study demonstrates the remarkable variation in the immune recognition patterns of Venezuelan patients with either PTB or EPTB. It also recommends further evaluation of the usefulness of the serodiagnosis of TB. Tests using ESAT-6 and Ag85A synthetic peptides demonstrated that a combinatorial use of different ESAT-6 and Ag85A epitopes could provide a screening strategy for the development of a multi-antigen ELISA. The higher sensitivities that were observed with the 12033/12034 and 11005/11006 combinations of peptides could reduce the frequency of false-negative results. False-positive results could be reduced using the peptide 29878 test, which achieved maximum specificities of $97.4 \%$ and $96.4 \%$. These techniques represent an opportunity to reduce the time-to-diagnosis for TB infection in regions that are endemic for TB infection in Venezuela.

\section{ACKNOWLEDGEMENTS}

To Mycobacteria Research Laboratories, Department of Microbiology, Immunology and Pathology, Colorado State University, USA (contract HHSN266200400091C/ADB contract NO1-AI-40091), for procuring the antigen 85 complex.

\section{REFERENCES}

Abebe F, Holm-Hansen C, Wilker HG, Bjune G 2007. Progress in serodiagnosis of Mycobacterium tuberculosis infection. Scand $J$ Immunol 66: 176-191.

Aoe K, Hiraki A, Murakami T, Eda R, Maeda T, Seguí K 2003. Diagnostic significance of interferon-gamma in tuberculous pleural effusions. Chest 123: 740-744.

Araujo Z, de Waard JH, de Larrea CF, López D, Fandiño C, Maldonado A, Hernández E, Ocaña Y, Ortega R, Singh M, Ottenhoff THM, Arend SM, Convit J 2004. Study of the antibody response against Mycobacterium tuberculosis antigens in Warao Amerindian children in Venezuela. Mem Inst Oswaldo Cruz 99: 517-524.

Araujo Z, Giampietro F, Cançado LC, Singh M, Wide A 2008. Comparison of serological responses in two different populations with pulmonary tuberculosis. Mem Inst Oswaldo Cruz 103: 661-667.

Arnadottir T, Rieder HI, Trebuq A, Waaler H 1996. Guidelines for conducting tuberculin skin test surveys in high prevalence countries. Tuber Lung Dis 77: 1-19.

Chiang IH, Sou J, Bai KJ, Lin TP, Luh KT, Yu CJ, Yang PC 1997. Serodiagnosis of tuberculosis. A study comparing three specific mycobacterial antigens. Am J Respir Crit Care Med 156: 906-911.

Conde MB, Suffys P, Lapa e Silva JR, Kritski AL, Dorman SE 2004. Immunoglobulin $\mathrm{A}(\mathrm{Ig} \mathrm{A})$ and $\operatorname{IgG}$ immune responses against p-90 antigen for diagnosis of pulmonary tuberculosis and screening for Mycobacterium tuberculosis infection. Clin Diagn Lab Immunol 11: 94-97.

Cunningham BC, Wells JA 1993. Comparison of a structural and a functional epitope. $J$ Mol Biol 234: 554-563.
Demkow U, Ziólkowski J, Filewska M, Białas-Chromiec B, Zielonka T, Michałowska-Mitczuk D, Kuś J, Augustynowicz E, Zwolska Z, Skopińska-Rózewska E, Rowińska-Zakrzewska E 2004. Diagnostic value of different serological tests for tuberculosis in Poland. J Physiol Pharmacol 55: 57-66.

Feng TT, Shou CM, Shen L, Qian Y, Wu ZG, Fan J, Zhang YZ, Tang YW, Wu NP, Lu HZ, Yao HP 2011. Novel monoclonal antibodies to ESAT-6 and CFP-10 antigens for ELISA-based diagnosis of pleural tuberculosis. Int J Tuberc Lung Dis 15: 804-810.

Fernández de Larrea C, Giampietro F, Luna L, Singh M, de Waard JH, Araujo Z 2011. Diagnosis accurancy of immunological methods in patients with tuberculosis pleuritis from an area with a high incidence of tuberculosis in Venezuela. Invest Clin 52: 23-34.

Gupta S, Shende N, Bhatia AS, Kumar S, Harinath BC 2005. IgG subclass antibody response to mycobacterial serine protease at different stages of pulmonary tuberculosis. Med Sci Monit 11: 585-588.

Harboe M 1998. B-cell epitopes and quantification of the ESAT-6 protein of Mycobacterium tuberculosis. Infect Immun 66: 717-723.

He XY, Li J, Hao J, Chen HB, Zhao YZ, Huang XY, He K, Xiao L, Ye LP, Qu YM, Ge LH 2011. Assessment of five antigens from Mycobacterium tuberculosis for serodiagnosis of tuberculosis. Clin Vaccine Immunol 18: 565-570.

Honore S, Vincensini JP, Hocqueloux L, Noguera ME, Farge D, Lagrange P, Herrmann JL 2001. Diagnostic value of a nested polymerase chain reaction assay on peripheral blood mononuclear cells from patients with pulmonary and extra-pulmonary tuberculosis. Int J Tuberc Lung Dis 5: 754-762.

Houghten RA 1985. General method for the rapid solid-phase synthesis of large numbers of peptides: specificity of antigen-antibody interaction at the level of individual amino acids. Proc Natl Acad Sci USA 82: 5131-5135.

Ireton GC, Greenwald R, Liang H, Esfandiari J, Lyashchenko KP, Reed SG 2010. Identification of Mycobacterium tuberculosis antigens of high serodiagnostic value. Clin Vaccine Immunol 17: 1539-1547.

Kashyap RS, Rajan AN, Ramteke SS, Agrawal VS, Kelkar SS, Purohit HJ, Taori GM, Daginawala HF 2007. Diagnosis of tuberculosis in an Indian population by an indirect ELISA protocol based on detection of antigen 85 complex: a prospective cohort study. BMC Infect Dis 7: 74-79.

Kudoh S, Kudoh T 1974. A simple technique for culturing tubercle bacilli. Bull World Health Organ 51: 71-82.

Kumar G, Dagur PK, Singh M, Yadav VS, Dayal R, Singh HB, Katoch VM, Sengupta U, Joshi B 2008. Diagnostic potential of $\mathrm{Ag} 85 \mathrm{C}$ in comparison to various secretory antigens for childhood tuberculosis. Scand J Immunol 68: 177-183.

López-Vidal Y, de Leon-Rosales SP, Castanon-Arreola M, RangelFrausto MS, Melendez-Herrada E, Sada-Diaz E 2004. Response of IFN-gamma and IgG to ESAT- 6 and $38 \mathrm{kDa}$ recombinant proteins and their peptides from Mycobacterium tuberculosis in tuberculosis patients and asymptomatic household contacts may indicate possible early-stage infection in the latter. Arch Med Res 35: 308-317.

Lyashchenko K, Colangeli R, Houde M, Al Jahdali H, Menzies D, Gennaro ML 1998. Heterogeneous antibody responses in tuberculosis. Infect Immun 66: 3936-3940.

Mabey D, Peeling RW, Ustianowski A, Perkins MD 2004. Diagnostics for the developing world. Nat Rev Microbiol 2: 231-240.

Merrifield RB 1963. Solid-phase peptide synthesis. I. The synthesis of a tetrapeptide. J Am Chem Soc 85: 2149-2154.

MSDS - Ministerio de Salud y Desarrollo Social 2006. Evaluación del Programa Nacional de control de la Tuberculosis. Año Evaluado 2006, MSDS, Caracas, 200 pp. 
Pottumarthy S, Wells VC, Morris AJ 2000. A comparison of seven tests for serological diagnosis of tuberculosis. J Clin Microbiol 38: $2227-2231$

Raja A, Uma Devi KR, Ramalingam B, Brennan PJ 2002. Immunoglobulin $\mathrm{G}, \mathrm{A}$ and $\mathrm{M}$ responses in serum and circulating immune complex elicited by the 16-kilodalton antigen of tuberculosis. Clin Diagn Lab Immunol 9: 308-312.

Ridley DS, Jopling WH 1966. Classification of leprosy according to immunity; a five-group system. Int J Lepr 34: 255-273.

Steingart KR, Dendukuri N, Henry M, Schiller I, Nahid P, Hopewell PC, Ramsay A, Pai M, Laal S 2009. Performance of purified antigens for serodiagnosis of pulmonary tuberculosis: a meta-analysis. Clin Vaccine Immunol 16: 260-276.

Tam JP, Heath WF 1983. SN1 and SN2 mechanisms for the deprotection of synthetic peptides by hydrogen fluoride. Studies to minimize the tyrosine alkylation side reaction. Int J Pept Protein Res 21: 57-65.

Tiwari RP, Hattikudur NS, Bharmal RN, Kartikeyan S, Deshmukh NM, Bisen PS 2007. Modern approaches to a rapid diagnosis of tuberculosis: promises and challenges ahead. Tuberculosis (Edinb) 87: 193-201.

Turneer M, Van Vooren JP, De Bruyn J, Serruys E, Dierckx P, Yernault J 1998. Humoral immune response in human tuberculosis: immunoglobulins $\mathrm{G}, \mathrm{A}$ and $\mathrm{M}$ directed against the purified
P32 protein antigen of Mycobacterium bovis bacillus CalmetteGuérin. J Clin Microbiol 26: 1714-1719.

Valdes L, San José E, Alvarez D, Sarandeses A, Pose A, Chomón B, Alvarez Dobaño JM, Salgueiro M, Rodríguez Suárez JR 1993. Diagnosis of tuberculous pleurisy using the biologic parameters adenosine deaminase, lysozyme and interferon gamma. Chest 103: $458-465$.

Van Regenmortel MHV 2001. Antigenicity and immunogenicity of synthetic peptides. Biologicals 29: 209-213.

Wallis RS, Perkins M, Phillips M, Joloba M, Demchuk B, Namale A, Johnson JL, Williams D, Wolski K, Teixeira L, Dietze R, Mugerwa RD, Eisenach K, Ellner JJ 1998. Induction of the antigen 85 complex of Mycobacterium tuberculosis in sputum: a determinant of out-come in pulmonary tuberculosis treatment. J Infect Dis 178: 1115-1121.

Weldingh K, Rosenkrands I, Okkels LM, Doherty TM, Andersen P 2005. Assessing the serodiagnostic potential of 35 Mycobacterium tuberculosis proteins and identification of four novel serological antigens. J Clin Microbiol 43: 57-65.

WHO - World Health Organization 2009. Global tuberculosis control: surveillance, planning, financing, WHO, Geneva, $376 \mathrm{pp}$.

Wiker HG, Harboe M 1992. The antigen 85 complex: a major secretion product of Mycobacterium tuberculosis. Microbiol Rev 56: 648-661 